\title{
Synthesis and characterisation of several di-, tri-, and tetra- radicals linked by flexible or rigid linkers
}

\author{
Miron T. Caproiu, ${ }^{a}$ Gabriela Ionita, ${ }^{b}$ Constantin Draghici, ${ }^{a}$ and Petre Ionita*b \\ ${ }^{a}$ Center of Organic Chemistry, Spl. Independente 202B, Bucharest 71141, Romania \\ ${ }^{b}$ Institute of Physical-Chemistry, 202 Spl. Independentei, Bucharest 060021, Romania \\ E-mail:pionita@icf.ro
}

\begin{abstract}
This paper reports the synthesis of eight new polyradicals (di-, tri-, and tetra- stable radicals) linked by flexible (aliphatic) or rigid (aromatic) units. Their preparation, as well as their electron paramagnetic resonance characteristics are described and discussed. These polyradicals were successfully tested as spin-labels for gold nanoparticles.
\end{abstract}

Keywords: ESR, stable radical, polyradicals, synthesis, gold nanoparticles

\section{Introduction}

Polyradicals are compounds of special interest, due to their (para)magnetic properties, as well as their potential probes and sensors in many physical, chemical, or biological processes, or in materials labelling (like nanoparticles, silica, polymers, etc).$^{1-4}$

Organic-based magnetic materials are an attractive field in materials chemistry, which offers the possibility to build step-by-step single molecules which contain many stable radical moieties, of the same type or of different types. ${ }^{5-8}$ Co-operative magnetic properties may led to the formation of an organo-metallic solid, with important practical properties; moreover, the properties of these compounds may be tuned by using the appropriate building blocks to get it right. $^{9-11}$

Pure organic materials based on stable polyradicals (stable organic high spin molecules) are shown in literature data, and they exhibit many interesting features, like ferro- or ferric magnetism, molecular magnets or wires, switches, and other metallic properties. ${ }^{5-11}$

Our interest ${ }^{12}$ in preparation of new paramagnetic (multispin) compounds prompted us to synthesise several stable di-, tri-, and tetra-radicals, in which the paramagnetic moieties are separated by flexible or rigid linkers. Due to their constituency, these polyradicals may be involved in several types of processes, like acid-base or redox, or to label gold nanoparticles. ${ }^{4-6} \mathrm{~T}$

There is also an interest in measuring and in the correlation of the distances between spins 
with the properties of the polyradicals. Measuring molecular scale distances is a topic in some biological systems, in which X-ray spectroscopy cannot be applied. Knowledge of the electronic, redox, and distances between spins is necessary to predict the final properties of the magnetic material, and the electron paramagnetic resonance (EPR or ESR) spectroscopy is a fast and reliable tool, from which the (para)magnetic properties are easily extracted. The distances between spins in a polyradical system greatly affect the shape of ESR spectra (because an exchange interaction appears between two or more vicinal spins). ${ }^{1,2}$

An ESR spectrum is usually characterised by the hyperfine coupling constants $a$, the $g$ value, and the exchange interaction $J{ }^{1,2}$ Thus, if the distance between two spins is large, the ESR spectra of the system looks like two superimposed ESR spectrum $(J<<a)$, if the distance is short enough that the two unpaired electrons start to 'feel' each other (because they act as a small magnets), the corresponding spectra have a very complicated shape, with supplementary lines between and outside the normal spectra lines $(J \sim a)$, or, in the last case, if the distance is very short, a strong interaction appears between the two unpaired electrons, and in the ESR spectra characteristic supplementary lines appears in the middle of the normal ones. ${ }^{1,2}$

In this paper we report the synthesis and characterisation of several stable polyradicals (Fig. 1). All of them have been tested as spin-labels for gold nanoparticles, as described below.

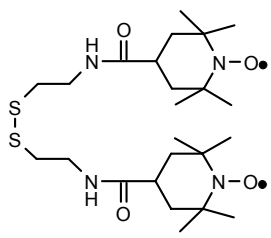

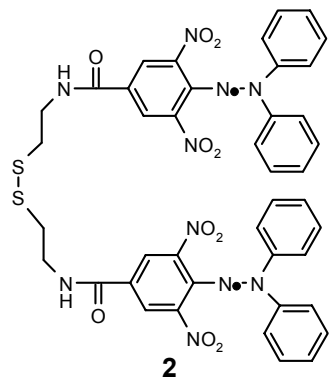

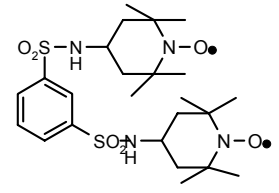

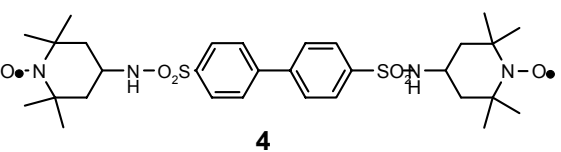

4

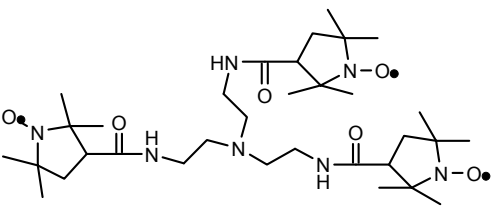

7

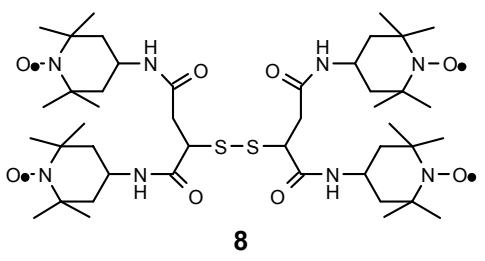

Figure 1. Chemical structure of the new polyradicals studied in this paper. 


\section{Experimental Section}

General Procedures. All solvents and chemicals were supplied by Aldrich or Chimopar, and used as received. The UV-Vis spectra were measured on a UVD-3500 spectrometer; the NMR spectra were measured on a Varian Gemini 300BB spectrometer; the ESR spectra were measured in DCM at ambient temperature $(295-300 \mathrm{~K})$ on a Jeol Jes FA100 spectrometer, using the following general settings: centre field $3330 \mathrm{G}$, sweep field $100 \mathrm{G}$, frequency $9.42 \mathrm{GHz}$, power 1 $\mathrm{mW}$, sweep time $60 \mathrm{~s}$, time constant $0.1 \mathrm{~s}$, modulation frequency $100 \mathrm{kHz}$, gain 100 , and modulation width 1 G. 4-(N,N-diphenylhydrazine)-3,5-dinitrobenzoic acid was synthesised as previously described. ${ }^{13}$ Phosphine protected gold nanoparticles were synthesised as previously described. ${ }^{3}$

1. To $1 \mathrm{mmol}$ cystamine dissolved in DCM was added $2.2 \mathrm{mmol} 4$-carboxytempo and $2.2 \mathrm{mmol}$ DCC, and the reaction mixture left overnight. Next day the DCM solution was filtered, washed with aqueous hydrochloric acid $(1 \mathrm{~N})$, aqueous sodium hydroxide $(1 \mathrm{~N})$, water, and dried over anhydrous sodium sulphate. Removal of the solvent under vacuum (at no more than $40^{\circ} \mathrm{C}$ ) afford then crude product, which was column chromatographied on silica gel, using $\mathrm{DCM} /$ methanol $9 / 1(\mathrm{v} / \mathrm{v})$ as eluent. The final yield was $\sim 65 \%$. Molecular formula $\mathrm{C}_{24} \mathrm{H}_{44} \mathrm{~N}_{4} \mathrm{O}_{4} \mathrm{~S}_{2}, \quad \mathrm{M}=516$, calculated elemental CHN composition (\%): $\mathrm{C}=55.78, \mathrm{H}=8.58$, $\mathrm{N}=10.84$; found $\mathrm{C}=55.65, \mathrm{H}=8.59, \mathrm{~N}=10.24$. ESI-MS $515(-\mathrm{H}, 100 \%)$.

2. To $1 \mathrm{mmol}$ cystamine dissolved in DCM was added $2.2 \mathrm{mmol} 4$-(N,N-diphenylhydrazine)3,5dinitrobenzoic acid- and $2.2 \mathrm{mmol}$ DCC, and the reaction mixture left for three days. The DCM solution was filtered off, washed with aqueous hydrochloric acid (1 N), aqueous sodium hydroxide (1 N), water, and dried over anhydrous sodium sulphate. Removal of the solvent under vacuum (at no more than $40^{\circ} \mathrm{C}$ ) afford then crude product, which was column chromatographied on silica gel, using benzene/ethyl acetate $9 / 1(\mathrm{v} / \mathrm{v})$ as eluent. ${ }^{1} \mathrm{H}-\mathrm{NMR}\left(\mathrm{CDCl}_{3}, \delta \mathrm{ppm}, J \mathrm{~Hz}\right)$ : 9.84 (s, 1H, HN- $\beta$, deuterable); 8.82 (vls, $1 \mathrm{H}, \mathrm{H}-11$ or H-9); 8.30 (vls); 7.81 (bt, $1 \mathrm{H}, \mathrm{HN}-\gamma$ ); 7.29 (dd, 4H, H-3-5-3'5', 7.4, 8.5); 7.13 (tt, 2H, H-4-4', 1.2, 7.4); 7.10 (dd, 4H, H-2-6-2'-6', 1.2, 8.5); 3.72 (q, 2H, H-14, 6.3); 2.92 (t, 2H, H-15, 6.3). ${ }^{13} \mathrm{C}-\mathrm{NMR}\left(\mathrm{CDCl}_{3}, \delta \mathrm{ppm}\right.$ ): 163.79 (C-13); 146.44 (C-1-1'); 140.32 (C-8-12); 134.20 (C-7); 123.23 (C-10); 130.00 (bs, C-9 or C-11); 129.10 (bs, C-11 or C-9); 129.42 (C-3-5-3'-5'); 125.38 (C-4-4'); 120.48 (C-2-6-2'-6'); 39.80 (C-14); 37.68 (C-15). Oxidation of the hydrazine in DCM with excess lead dioxide affords the corresponding diradical, with the final yield $\sim 50 \%$. Molecular formula $\mathrm{C}_{42} \mathrm{H}_{32} \mathrm{~N}_{10} \mathrm{O}_{10} \mathrm{~S}_{2}, \mathrm{M}=900$, calculated elemental CHN composition (\%): $\mathrm{C}=55.99, \mathrm{H}=3.58, \mathrm{~N}=15.54$; found $\mathrm{C}=55.77, \mathrm{H}=$ 3.75, N=15.38. ESI-MS $901(+\mathrm{H}, 100 \%)$. UV-Vis $(\mathrm{DCM}): \lambda_{\max }=512 \mathrm{~nm}$ (the corresponding hydrazine has $\lambda_{\max }=392$, and in basic media $\lambda_{\max }=635 \mathrm{~nm}$ ).

3. To $1 \mathrm{mmol}$ of benzene-1,3-disulfonyl chloride dissolved in DCM was added $2.2 \mathrm{mmol}$ of 4 aminotempo and $5 \mathrm{~mL}$ of pyridine, and the mixture left till next day. The mixture was washed with aqueous hydrochloric acid (1 N), aqueous sodium hydroxide $(1 \mathrm{~N})$, water, and dried over anhydrous sodium sulphate. Removal of the solvent under vacuum (at no more than $40^{\circ} \mathrm{C}$ ) 
afford then crude product, which was column chromatographied on silica gel, using DCM/ether $7 / 3(\mathrm{v} / \mathrm{v})$ as eluent. The final yield was $\sim 75 \%$. Molecular formula $\mathrm{C}_{24} \mathrm{H}_{40} \mathrm{~N}_{4} \mathrm{O}_{6} \mathrm{~S}_{2}, \mathrm{M}=544$, calculated elemental $\mathrm{CHN}$ composition (\%): $\mathrm{C}=52.91, \mathrm{H}=7.40, \mathrm{~N}=10.29$; found $\mathrm{C}=52.55$, $\mathrm{H}=7.33$, $\mathrm{N}=9.55$. ESI-MS 544 (100\%).

4. To $1 \mathrm{mmol}$ of biphenyl-4,4'-disulfonyl chloride dissolved in DCM was added $2.2 \mathrm{mmol}$ of 4aminotempo and $5 \mathrm{~mL}$ of pyridine, and the mixture left till next day. The mixture was washed with aqueous hydrochloric acid $(1 \mathrm{~N})$, aqueous sodium hydroxide $(1 \mathrm{~N})$, water, and dried over anhydrous sodium sulphate. Removal of the solvent under vacuum (at no more than $40^{\circ} \mathrm{C}$ ) afford then crude product, which was column chromatographied on silica gel, using DCM/ether $7 / 3(\mathrm{v} / \mathrm{v})$ as eluent. The final yield was $\sim 65 \%$. Molecular formula $\mathrm{C}_{30} \mathrm{H}_{44} \mathrm{~N}_{4} \mathrm{O}_{6} \mathrm{~S}_{2}, \mathrm{M}=620$, calculated elemental $\mathrm{CHN}$ composition (\%): $\mathrm{C}=58.04, \mathrm{H}=7.14, \mathrm{~N}=9.02$ : found $\mathrm{C}=57.78, \mathrm{H}=7.10$, $\mathrm{N}=8.27$. ESI-MS 620 (100\%).

5. To $1 \mathrm{mmol}$ of dibenzofuran-2,8-disulfonyl chloride dissolved in DCM was added $2.2 \mathrm{mmol}$ of 4-aminotempo and $5 \mathrm{~mL}$ of pyridine, and the mixture left till next day. The mixture was washed with aqueous hydrochloric acid (1 N), aqueous sodium hydroxide (1 N), water, and dried over anhydrous sodium sulphate. Removal of the solvent under vacuum (at no more than $40^{\circ} \mathrm{C}$ ) afford then crude product, which was column chromatographied on silica gel, using DCM/ether $7 / 3(\mathrm{v} / \mathrm{v})$ as eluent. The final yield was $\sim 65 \%$. Molecular formula $\mathrm{C}_{30} \mathrm{H}_{42} \mathrm{~N}_{4} \mathrm{O}_{7} \mathrm{~S}_{2}, \mathrm{M}=634$, calculated elemental $\mathrm{CHN}$ composition (\%): $\mathrm{C}=56.76, \mathrm{H}=6.66, \mathrm{~N}=8.83$; found $\mathrm{C}=56.56, \mathrm{H}=6.60$, $\mathrm{N}=8.23$. ESI-MS 634 (100\%).

6. To $1 \mathrm{mmol}$ of benzene-1,3,5-tricarbonyl chloride dissolved in DCM was added $3.3 \mathrm{mmol}$ of 4aminotempo and $5 \mathrm{~mL}$ of pyridine, and the mixture left till next day. The mixture was washed with aqueous hydrochloric acid (1 N), aqueous sodium hydroxide (1 N), water, and dried over anhydrous sodium sulphate. Removal of the solvent under vacuum (at no more than $40^{\circ} \mathrm{C}$ ) afford then crude product, which was column chromatographied on silica gel, using $\mathrm{DCM} /$ methanol $9 / 1(\mathrm{v} / \mathrm{v})$ as eluent. The final yield was $\sim 80 \%$. Molecular formula $\mathrm{C}_{36} \mathrm{H}_{57} \mathrm{~N}_{6} \mathrm{O}_{6}$, $\mathrm{M}=669$, calculated elemental $\mathrm{CHN}$ composition (\%): $\mathrm{C}=64.54, \mathrm{H}=8.57, \mathrm{~N}=12.55$; found $\mathrm{C}=64.37, \mathrm{H}=8.55, \mathrm{~N}=12.44$. ESI-MS $668(-\mathrm{H}, 100 \%)$.

7. To $1 \mathrm{mmol}$ of $\mathrm{N}$-(triaminoethyl)amine dissolved in DCM was added $4 \mathrm{mmol}$ of 4carboxyproxyl free radical and $5 \mathrm{mmol}$ of EEDQ, and the mixture left for 5 days. The mixture was washed with aqueous hydrochloric acid $(1 \mathrm{~N})$, aqueous sodium hydroxide $(1 \mathrm{~N})$, water, and dried over anhydrous sodium sulphate. Removal of the solvent under vacuum (at no more than $40^{\circ} \mathrm{C}$ ) afford then crude product, which was column chromatographied on silica gel, using $\mathrm{DCM} /$ methanol $9 / 1(\mathrm{v} / \mathrm{v})$ as eluent. The final yield was $\sim 50 \%$. Molecular formula $\mathrm{C}_{33} \mathrm{H}_{60} \mathrm{~N}_{7} \mathrm{O}_{6}$, $\mathrm{M}=650$, calculated elemental $\mathrm{CHN}$ composition $(\%)$ : $\mathrm{C}=60.89, \mathrm{H}=9.29, \mathrm{~N}=15.06$; found $\mathrm{C}=$ 60.81, $\mathrm{H}=9.33, \mathrm{~N}=14.48$. ESI-MS $650(100 \%)$.

8. Oxidation of 2-mercaptosuccinic acid afforded the corresponding disulphide: ${ }^{1} \mathrm{H}-\mathrm{NMR}$ (DMSO-d ${ }_{6}, \delta$ ppm, $J$ Hz): 12.70 (bs, 1H, HOOC, deuterable); 3.78 (dd, 1H, H-2, 5.1, 9.2); 3.75 $(\mathrm{dd}, 1 \mathrm{H}, \mathrm{H}-2,5.1,9.2) ; 2.83(\mathrm{dd}, 1 \mathrm{H}, \mathrm{H}-3 \mathrm{~A}, \mathrm{AB}$ system, 9.2, 17.0); 2.82(dd, 1H, H-3A, AB system, 9.2, 17.0); 2.70 (dd, 2H, H-3B, 5.2, 17.0). ${ }^{13} \mathrm{C}-\mathrm{NMR}$ (DMSO-d 6 , $\delta$ ppm): 171.67 (2C-4); 
171.60 (1C-1); 171.49 (1C-1); 48.22 (C-2); 48.06 (C-2); 47.63 (C-2); 47.45 (C-2); 36.04 (2C-3); 35.80 (2C-3). $1 \mathrm{mmol}$ of the disulphide dissolved in a mixture of DCM and THF was reacted for five days with $5 \mathrm{mmol}$ of 4 -aminotempo in the presence of $5 \mathrm{mmol}$ EEDQ. The mixture was washed with aqueous hydrochloric acid $(1 \mathrm{~N})$, aqueous sodium hydroxide $(1 \mathrm{~N})$, water, and dried over anhydrous sodium sulphate. Removal of the solvent under vacuum (at no more than $40^{\circ} \mathrm{C}$ ) afford then crude product, which was column chromatographied on silica gel, using DCM/ethyl acetate $9 / 1(\mathrm{v} / \mathrm{v})$ as eluent. The final yield was $\sim 55 \%$. Molecular formula $\mathrm{C}_{44} \mathrm{H}_{78} \mathrm{~N}_{8} \mathrm{O}_{8} \mathrm{~S}_{2}$, $\mathrm{M}=910$, calculated elemental $\mathrm{CHN}$ composition (\%): $\mathrm{C}=57.99, \mathrm{H}=8.62, \mathrm{~N}=12.30$; found $\mathrm{C}=$ 57.90, $\mathrm{H}=8.65, \mathrm{~N}=11.88$. ESI-MS $933(+\mathrm{Na}, 100 \%)$.

Testing polyradicals 1-8 as spin-labels for gold nanoparticles. To a $0.1 \mathrm{~mL}$ solution of triphenylphosphine protected gold nanoparticles ${ }^{3}\left(10^{-4} \mathrm{M}\right.$, in DCM) has been added $0.1 \mathrm{~mL}$ solution of newly synthesised radicals $\mathbf{1 - 8}\left(10^{-4} \mathrm{M}\right.$, in DCM), and the mixture analyzed by ESR spectroscopy.

\section{Results and Discussion}

Synthesis. All the polyradicals shown in Fig. 1 were easily obtained by standard coupling procedures (Scheme 1), and their purity checked by TLC (single spot). The coupling procedures involved the reactions between an amine with a carboxylic acid in the presence of DCC, or with a carbonyl-chloride in the presence of Py, or with a sulfochloride in the presence of Py as well. Good yields were obtained, usually between $50-80 \%$. The separation of analytical sample from the reaction mixture was done by column chromatography on silica gel, with an appropriate mixture of solvents (see Experimental). The compounds are stable over months at room temperature, and no decomposition products were found after long time.

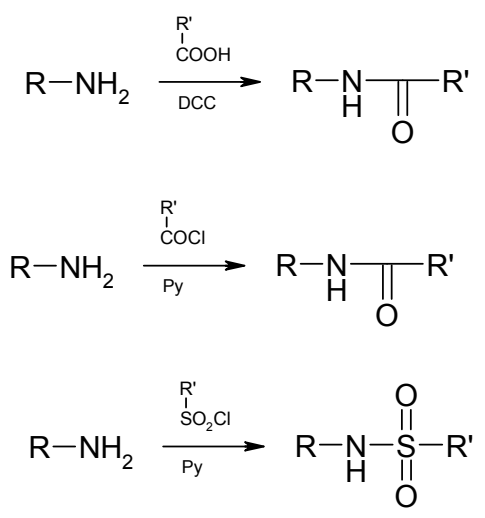

Scheme 1. General procedures used for the preparation of the polyradicals 1-8. 
ESR spectra. ESR spectra of polyradicals 1-8 are shown in Fig. 2 (the total length of the recorded ESR spectra is $100 \mathrm{G}$ ). These recorded ESR spectra can be divided into three types, according to the general classification. ${ }^{1}$ In all the spectra, besides compounds 6 and 7, the interaction between the spins is clearly visible, by apparition of the supplementary lines in ESR spectra (un unpaired electron will give three lines in the ESR spectrum, due to the vicinal nitrogen atom splitting, with the usual hyperfine coupling constant of $\sim 15 \mathrm{G}$ ). A special case is compound 2, which have two vicinal nitrogen atoms, with similar hyperfine coupling constants $(\sim 9 \mathrm{G})$, and hence a different shape of the spectrum is noticed.

For all the compounds 1-5 and $\mathbf{8}$, the presence of the middle low intensity lines between the expected triplets is a strong proves for the interacting spins. A slightly different case is observed also for compound 4, which showed supplementary splitting inside and outside the normal triplet; a careful examination of the ESR spectrum shows two very low intensity peaks outside the triplet. This case is the due to the condition $J \sim a$, in which the values for the nitrogen hyperfine coupling constants are of the same order of magnitude as the exchange interaction between the spins. ${ }^{1,2}$
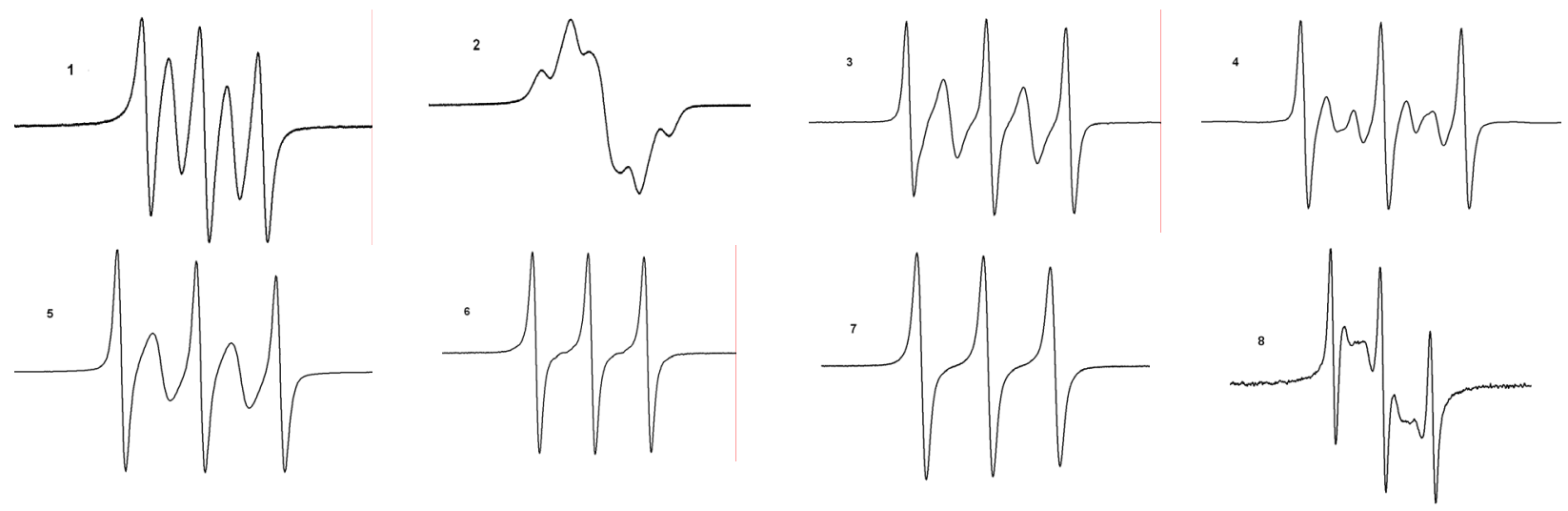

Figure 2. ESR spectra of the compounds 1-8.

Calculation of the interspin distances. In order to evaluate the distance between the radical moieties (interspin distance), we used the computational method delivered by the CHIMERA free software; ${ }^{14,15}$ thus, the average values measured for the compounds $\mathbf{1 - 8}$ were 6.69, 17.72, $13.01,11.83,8.43,13.35,8.50$, and $10.61 \mathrm{~A}^{\circ}$, respectively. As we can notice, for the compound 1, which has the smallest interspin distance $\left(6.69 \mathrm{~A}^{\mathrm{o}}\right)$, the interaction between spins is the highest, as seen by the ratio between the height of the middle lines (lines second and fourth, which appear only in the case of an interspin interaction) and the other lines (first, third and fifth lines, which characterize the common ESR spectrum). However, for the other compounds, there is no fixed rule regarding the correlation between the average distance provided by the molecular modelling software and the ESR spectra. 
Polyradicals 1-8 as spin-labels for gold nanoparticles. The affinity of gold for sulphurcompounds is very well know, and this has been extensively used for the spin-labelling of the gold nanoparticles. ${ }^{3}$ Thus, the simple mixing of gold nanoparticles with a disulphide diradical lead to the nanoparticles spin-labelling (the radical moiety is strongly attached on the gold surface, Scheme 2). This process is easily monitored by ESR, due to the changes which instantly are revealed in the spectrum shape. For all the compounds (except 6 and 7) the disappearance of the ESR middle lines (attributed to the interactions between adjacent spins) is noticed (Scheme 2). As a remark, even the sulphamido group in compounds 3-5 led to their attachment to the nanoparticles surface. In the case of the compounds 6 and 7, which do not shown the exchange middle lines, it is noticed the decreasing of the intensity of the last line (high-field), which also proved the attachment of the polyradicals on the nanoparticles surface.
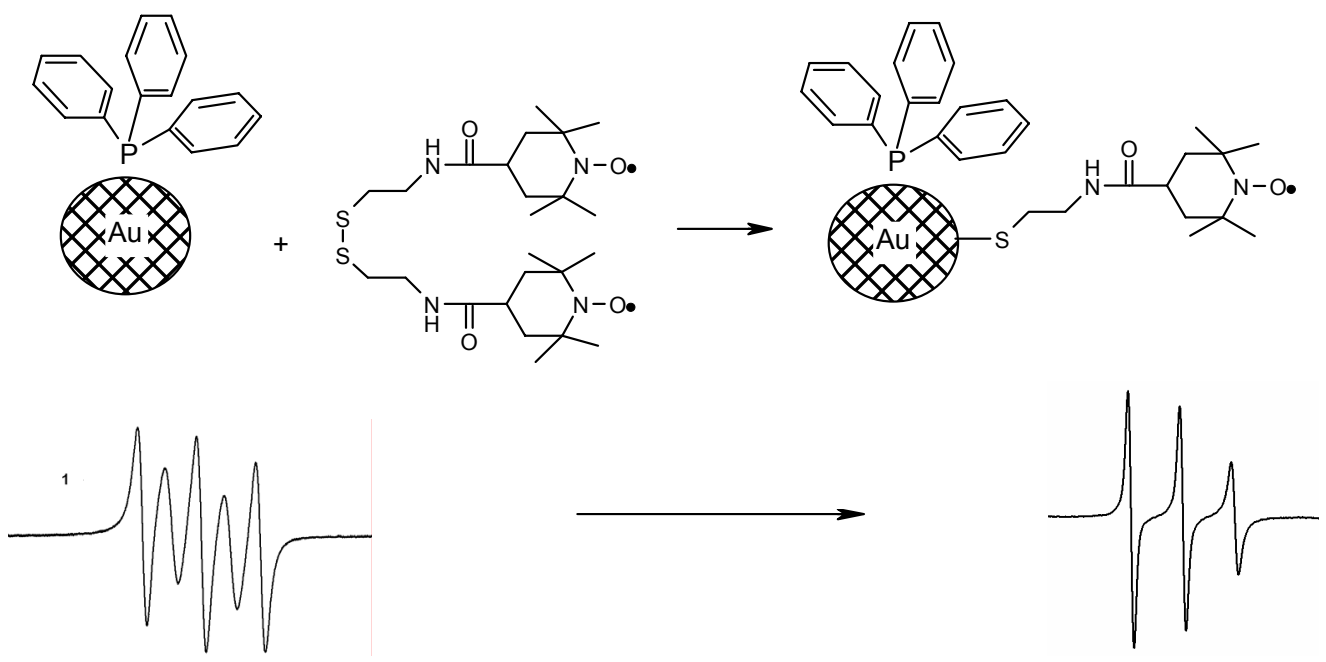

Scheme 2. Due to the attachment of the spin to gold nanoparticles, the ESR spectrum changes as shown.

\section{Conclusions}

ESR spectra demonstrated the synthesis of the stable free (poly)radicals 1-8; for most of them, the apparition of the supplementary lines besides the expected triplets clearly indicates the presence of spin-spin interaction. In the presence of gold nanoparticles, the spin-spin interaction disappears, proving the attachment of the radical moiety on the nanoparticles surface.

\section{Acknowledgements}

This work was partially sponsored by the CNCSIS PN2 'Idea’ 158/1.10.2007. 


\section{References}

1. Forrester, A. R. Organic Chemistry of Stable Free Radicals, Academic Press: London, 1968.

2. Borden, W. T. Diradicals, John Wiley: New York, 1982.

3. Ionita, P.; Gilbert, B. C.; Chechik, V. Angew. Chem. Int. Ed. 2005, 44, 3720 (and references herein).

4. Sahini, V. Em.; Ionita, G.; Ionita, P. Acta Chim. Slovenica 2000, 47, 111.

5. Constantinescu, T.; Ionita, P.; Chiorescu, I.; Ionita, G. Central Eur. J. Chem. 2003, 1, 465.

6. Ionita, P.; Whitwood, A. C.; Gilbert, B. C. J. Chem. Soc. Perkin Trans 2 2001, 1453.

7. Catala, L.; Moigne, J.; Gruber, N.; Novoa, J. J.; Rabu, P.; Belorizky, E.; Turek, P. Chem. Eur. J. 2005, 11, 2440.

8. Stroh, C.; Ziessel, R.; Raudaschl, S. G.; Kohler, F. H.; Turek, P. J. Mat. Chem. 2005, 15, 850.

9. Hirel, C.; Pecaut, J.; Choua, S.; Turek, P.; Amabilino, D. B.; Veciana, J.; Rey, P. Eur. J. Org. Chem. 2005, 2, 348.

10. Ziessel, R.; Stroh, C.; Heise, H.; Kohler, F. H.; Turek, P.; Claiser, N.; Souhassou, M.; Lecomte, C. J. Am. Chem. Soc. 2004, 126, 12604.

11. Catala, L.; Turek, P. J. Chim. Phys. 1999, 96, 1551.

12. Tudose, M.; Ionita, P.; Dumitrascu, F.; Draghici, C.; Caproiu, M. T.; Covaci, I. C.; Constantinescu, T.; Banciu, M. D.; Balaban, A. T. ARKIVOC 2005, (iv), 225.

13. Luca, C.; Ionita, P.; Caproiu, M. T.; Caldararu, H.; Constantinescu, T. Rev. Roum. Chem. 1998, 43, 221.

14. http://www.cgl.ucsf.edu/chimera/

15. Pettersen, E. F.; Goddard, T. D.; Huang, C. C.; Couch, G. S.; Greenblatt, D. M.; Meng, E. C.; Ferrin, T. E. J. Comput. Chem. 2004, 25, 1605. 